\title{
Spontaneous Regression of Blastic Plasmacytoid Dendritic Cell Neoplasm Following Sepsis by Serratia marcescens: A Case Report and Literature Review
}

\author{
Akane Suzuki $^{1}$, Shori Abe ${ }^{1}$, Kaori Koyama ${ }^{2}$, Shinju Suzuki ${ }^{2}$, Munenori Nagao ${ }^{2}$, \\ Masahiro Kobayashi ${ }^{1}$, Jun Nomura ${ }^{1}$, Tomomi Tsutsumi ${ }^{1}$, Tomoki Takeda ${ }^{1}$, Yumiko Oka ${ }^{1}$, \\ Yuko Shirota ${ }^{1}$, Naruhiko Takasawa ${ }^{1}$, Takao Kodera ${ }^{1}$, Yoko Okitsu ${ }^{3}$, Shinichiro Takahashi ${ }^{3}$, \\ Ryo Ichinohasama ${ }^{4}$ and Junichi Kameoka ${ }^{1}$
}

\begin{abstract}
:
Spontaneous regression is rare in patients with blastic plasmacytoid dendritic cell neoplasm (BPDCN). An 85-year-old man presented with pancytopenia and skin lesions, and the bone marrow exhibited $79.6 \%$ CD4+, CD56+, CD123+, and TCL-1+ abnormal cells, with a normal karyotype; he was thus diagnosed with BPDCN. While being followed without chemotherapy, he was admitted due to sepsis induced by Serratia marcescens, which was successfully treated with antibiotics. Notably, his blood cell counts improved, and the skin lesions disappeared. To our knowledge, this is the first reported case of spontaneous regression of BPDCN with a decrease in tumor cells in the bone marrow following sepsis.
\end{abstract}

Key words: spontaneous regression, blastic plasmacytoid dendritic cell neoplasm, sepsis, Serratia marcescens

(Intern Med 60: 927-933, 2021)

(DOI: 10.2169/internalmedicine.5820-20)

\section{Introduction}

Blastic plasmacytoid dendritic cell neoplasm (BPDCN) is a rare hematological malignancy that was first described in the 1990s as a new CD4+/CD56+ neoplasm $(1,2)$, and then established as a tumor derived from plasmacytoid dendritic cells in the 2000s $(3,4)$. BPDCN is characterized by agranular blastoid tumor cells with the positive expression of CD4, CD56, CD123 (IL-3 $\alpha$ receptor), CD303 (BDCA-2), and TCL-1 (5-7). In most cases, the skin is affected at the onset, followed by the infiltration of tumor cells to the bone marrow. Although chemotherapy can provide transient remission, the majority of cases eventually relapse and have a poor prognosis.

Spontaneous remission (SR) of cancer has long been described in various malignancies, including malignant mela- noma, neuroblastoma, nephroblastoma, and hematological malignancies $(8,9)$. Indeed, SR of acute leukemia due to infections was described as early as 1878 by Eisenlohr (10) and 1904 by Dock (11). Rashidi collected 46 reported cases of acute myeloid leukemia (AML) with SR (12), most of whom had a fever (91.3\%), largely due to pneumonia $(54.5 \%)$, and bacteremia (24.2\%). Although the precise mechanisms remain unclear, fever- or infection-induced immune reactivation/stimulation against malignant cells, with increased levels of cytokines, such as interleukin 1 (IL-1), tumor necrosis factor (TNF)- $\alpha$, and IL-12, and increased activity of natural killer (NK) cells and cytotoxic T-cells has been proposed (13-15).

SR in BPDCN is rare, partly due to the short history of the disease. To our knowledge, there have been only four reported cases of spontaneous remission of BPDCN (16-19) in the English literature, none of which were related to sepsis.

\footnotetext{
${ }^{1}$ Division of Hematology and Rheumatology, Tohoku Medical and Pharmaceutical University Hospital, Japan, ${ }^{2}$ Division of Palliative Care, Tohoku Medical and Pharmaceutical University Hospital, Japan, ${ }^{3}$ Department of Clinical Laboratory, Tohoku Medical and Pharmaceutical University Hospital, Japan and ${ }^{4}$ Division of Hematopathology, Tohoku University Hospital, Japan

Received for publication July 9, 2020; Accepted for publication August 25, 2020

Correspondence to Dr. Junichi Kameoka, j-kame@tohoku-mpu.ac.jp
} 
A

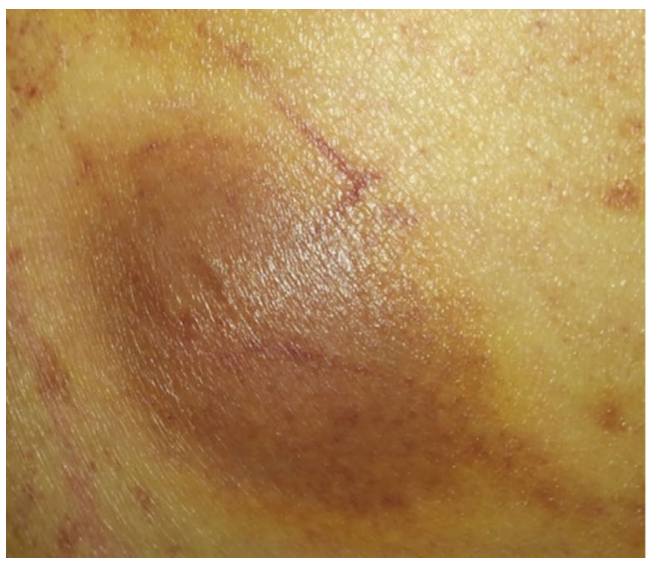

B

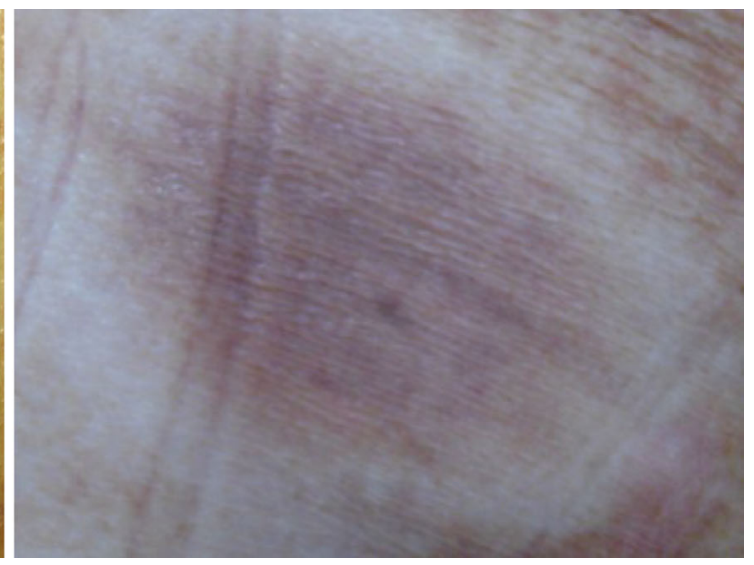

Figure 1. (A) Photograph showing one of two bruise-like or violaceous nodules on the patient's back at the first examination. (B) Photograph showing the complete diminishment of the nodule after recovery from sepsis.

A

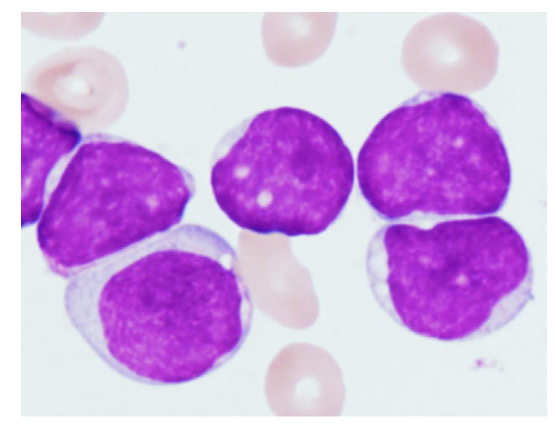

We herein report the first case of spontaneous regression of BPDCN, with a decrease in tumor cells in the bone marrow following severe sepsis induced by Serratia marcescens.

\section{Case Report}

An 85-year-old man visited the Tohoku Medical and Pharmaceutical University Hospital with shortness of breath in April 2019. He had a history of cancer of the appendix, which had undergone surgical resection six years previously without chemotherapy or radiotherapy. In addition, he had a history of aortic arch aneurysm, for which stent-graft treatment had been performed seven years previously.

The findings of a physical examination were negative, except for two skin nodules on the trunk (Fig. 1A) and signs of anemia in the palpebral conjunctivae. Initial blood cell counts showed severe pancytopenia as follows: White blood cell (WBC) count of $1.4 \times 10^{9} / \mathrm{L}$ with $8 \%$ abnormal cells, he- moglobin level of $5.1 \mathrm{~g} / \mathrm{dL}$, and platelets of $88 \times 10^{9} / \mathrm{L}$. Blood chemistry tests showed elevated serum creatinine $(1.57 \mathrm{mg} /$ $\mathrm{dL})$ and soluble interleukin-2 receptor $(922 \mathrm{U} / \mathrm{mL}$, normal: 157-474). Bone marrow aspirate showed hypercellular marrow with $79.6 \%$ small to medium-sized abnormal cells, with a high nucleus-to-cytoplasm ratio (Fig. 2A). Flow cytometry showed that the abnormal cells were CD4 dim+, CD56+, CD34+, HLA-DR+, GP-A+, CD1-, CD2-, CD3-, CD5-, CD10-, CD13-, CD14-, CD19-, CD20-, CD33-, and CD34(Fig. 2B). Immunohistochemistry of the bone marrow biopsy showed that the tumor cells were positive for CD4 dim, CD7, CD45, CD56 dim, CD123, TdT, Bcl6, and TCL1 , and negative for myeloperoxidase, CD2, CD3, CD5, CD8, CD10, CD68, CD79a, CD20, and CD79 (Fig. 3). A karyotype analysis showed no chromosomal abnormalities. A diagnosis of BPDCN was made, but no chemotherapy was given due to the advanced age and poor physical condition of the patient. He was discharged in late April and fol- 


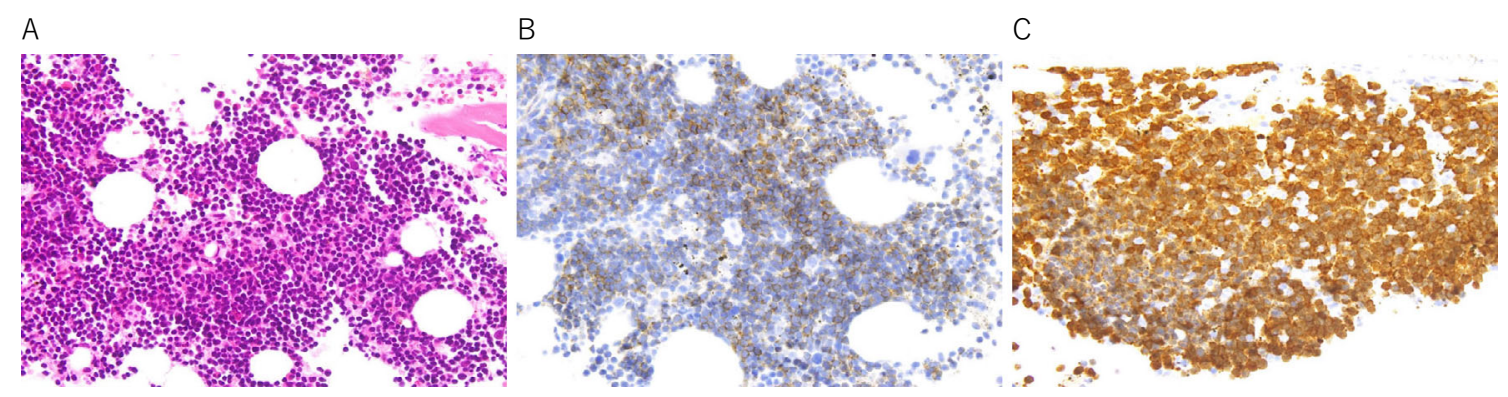

Figure 3. A bone marrow biopsy revealed (A) abnormal diffuse infiltration of tumor cells (Hematoxylin and Eosin staining, $\times 40$ ). An immunohistochemical analysis of the bone marrow biopsy revealed that the tumor cells were positive for (B) CD123 and (C) TCL-1.
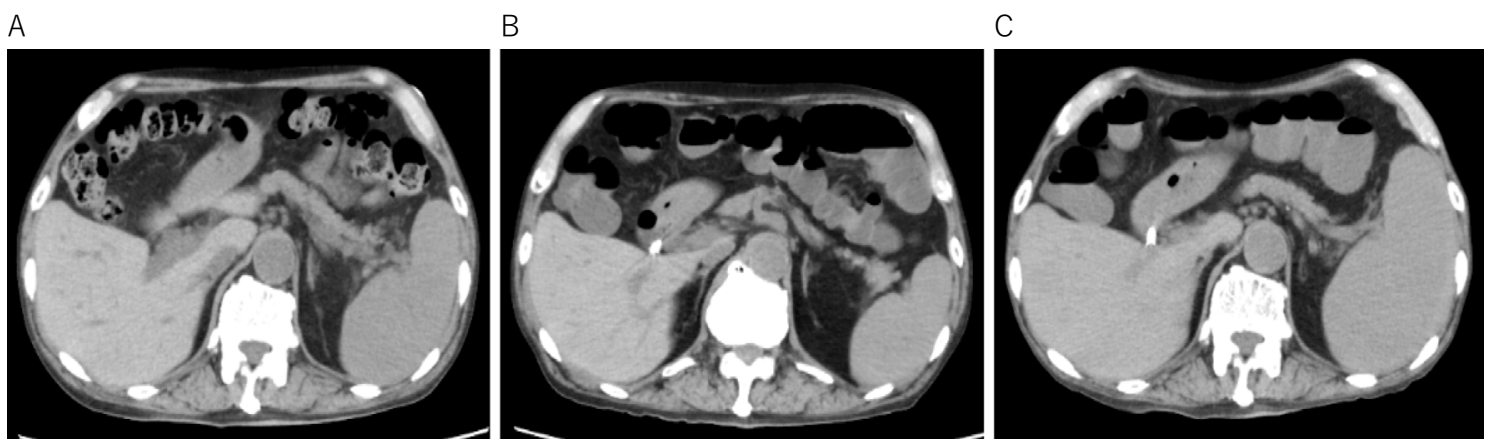

Figure 4. Sequential abdominal CT scans of the patient. (A) Abdominal CT in late June indicated mild splenomegaly. (B) Abdominal CT in late July indicated that the size of the spleen had decreased. (C) Abdominal CT in October indicated marked splenomegaly.

lowed up in the outpatient setting, receiving red blood cell transfusions.

In June, the patient was admitted to the hospital because of a high fever, up to $40^{\circ} \mathrm{C}$, accompanied by chills and shivering. He was hemodynamically unstable, with a systolic blood pressure of $103 \mathrm{mmHg}$ (ordinary systolic blood pressure, 130 to $150 \mathrm{mmHg}$ ). A physical examination revealed fine crackles in the lower parts of both lungs. Laboratory tests showed pancytopenia: WBC count, $0.3 \times 10^{9} / \mathrm{L}$, with $30.0 \%$ neutrophils; hemoglobin level, $5.0 \mathrm{~g} / \mathrm{dL}$; and a platelet count of $11 \times 10^{9} / \mathrm{L}$. Blood chemistry tests revealed increased levels of C-reactive protein, procalcitonin, and Ddimer, up to $17.53 \mathrm{mg} / \mathrm{dL}, 9.29 \mathrm{ng} / \mathrm{mL}$ (normal range $<0.5$ $\mathrm{ng} / \mathrm{mL}$ ), and $12.8 \mu \mathrm{g} / \mathrm{mL}$, respectively.

The Sequential Organ Failure Assessment score was 9 points (respiratory system +1 , nervous system 0 , cardiovascular system +1 , liver +2 , coagulation +4 , kidneys +1 ). The patient was diagnosed with sepsis, which was later supported by positive cultures of Serratia marcescens. He was treated with broad-spectrum antibiotics (tazobactam/piperacillin) combined with granulocyte-colony stimulating factor (G-CSF), which led to improvement after a week. Following the improvement of sepsis, the complete blood counts also improved spontaneously. His hemoglobin levels increased to greater than $10 \mathrm{~g} / \mathrm{dL}$ without transfusion, his absolute neutrophil counts increased to greater than $1,000 / \mu \mathrm{L}$ without G$\mathrm{CSF}$, and the platelet counts were normalized before being maintained around $150 \times 10^{9} / \mathrm{L}$ from late July to October. The size of the spleen was smaller than that observed on computed tomography (CT) performed a month earlier (Fig. 4A, B). In addition, the skin lesions disappeared, with only pigmentation remaining (Fig. 1B). The detailed clinical course is shown in Fig. 5.

Bone marrow aspiration performed in September showed a decrease in abnormal cells (29\%), while the peripheral blood showed no abnormal cells. The surface markers of the tumor cells in the bone marrow were basically the same as those at the initial bone marrow aspiration, except for a slightly higher expression of CD4 and negative expression of GPA (data not shown). The patient was considered to be in near-partial remission (PR), which we would call "spontaneous regression," according to the criteria of PR in AML (20).

The patient subsequently suffered from intestinal obstruction and pulmonary mycosis, which required surgical treatment and antifungal therapy, respectively. However, the platelet count gradually decreased, and tumor cells in the peripheral blood reappeared and increased, indicating the exacerbation of BPDCN (Fig. 5). A CT scan of the upper abdomen showed marked splenomegaly (Fig. 4C). In December 2019, he died of an uncontrollable infection, and no autopsy was performed. 

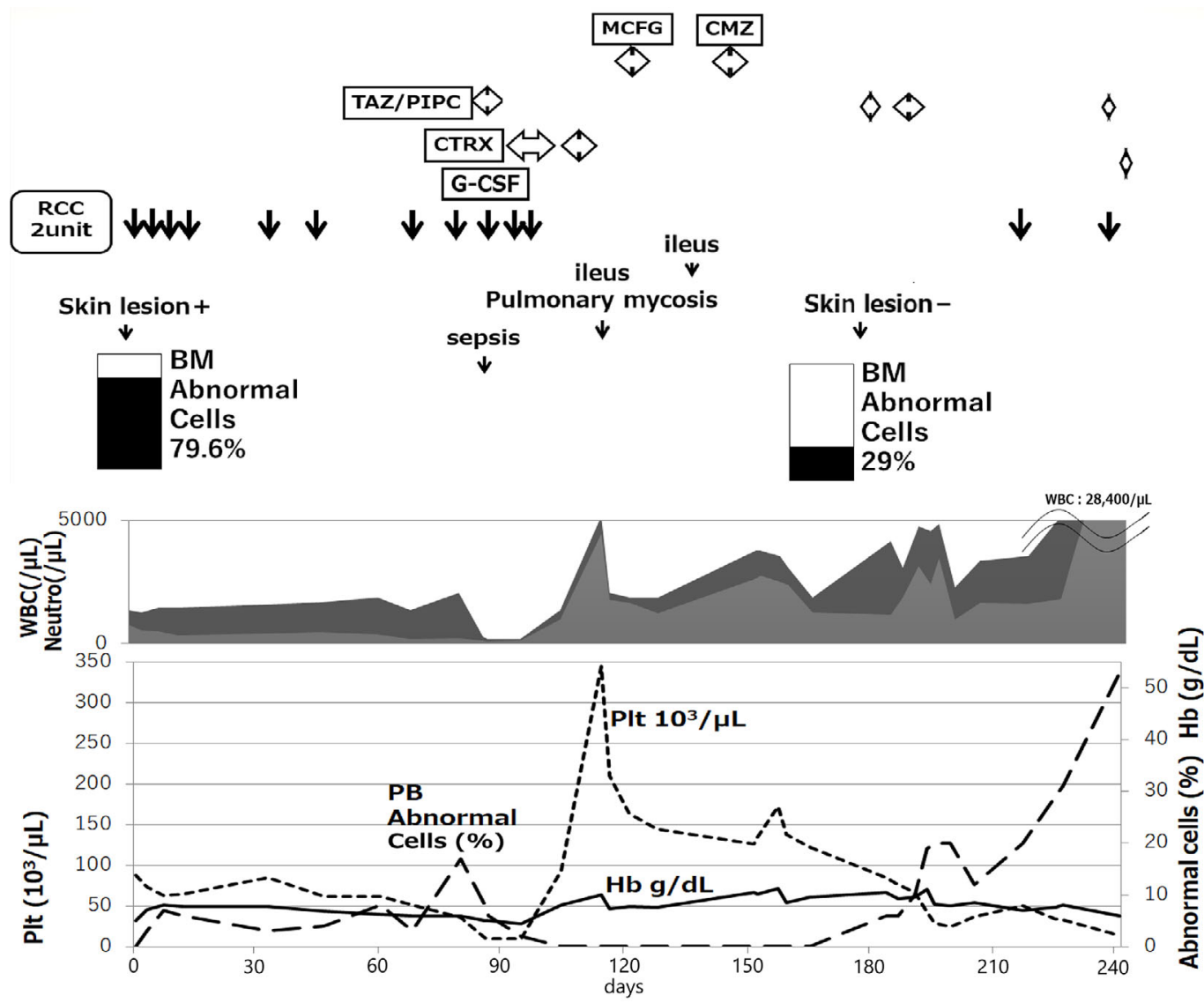

Figure 5. Clinical course of the patient. MCFG: micafungin, TAZ/PIPC: tazobactam/piperacillin, CTRX: ceftriaxone, G-CSF: granulocyte-colony stimulating factor, RCC: red cell concentrates, BM: bone marrow, PB: peripheral blood, WBC: white blood cell, Neutro: neutrophil, Plt: platelet count

\section{Discussion}

We herein report a rare case of spontaneous regression of BPDCN, probably the first case induced by severe sepsis. The diagnosis of BPDCN was based on the clinical features and bone marrow findings, including the positive expression of CD123 and TCL-1. Although the expression of CD4, particularly at the initial presentation, was rather weak, it does not exclude the diagnosis of BPDCN because CD4-negative BPDCN has been infrequently reported (21).

As the definition of PR of BPDCN has not yet been determined, we utilized the definition of remission in AML (20), which dictates that complete remission (CR) requires the following: 1) $<5 \%$ blasts in the bone marrow, with a count of at least 200 nucleated cells; 2) no blasts in the peripheral blood; 3) an absolute neutrophil count of more than $1,000 / \mu \mathrm{L}$; and 4) a platelet count of more than $100 \times 10^{9} / \mathrm{L}$. PR requires all hematologic values for CR but with a decrease of at least $50 \%$ in the percentage of blasts to $5 \%$ to $25 \%$ in the bone marrow. In this case, the absolute neutrophil count was $>1,000 / \mu \mathrm{L}$ without G-CSF, the platelet count was more than $100 \times 10^{9} / \mathrm{L}$, and peripheral blood exhibited no abnormal cells (see Fig. 5). Therefore, with the exception of the bone marrow findings, which showed $29 \%$ tumor cells, the current case met the criteria of PR.
To date, four cases of spontaneous remission of BPDCN have been reported (16-19) in the English literature, and we will review the five total cases, including our present one (Table). Four of the patients were men, and one was a woman. The patients ranged in age from 15 to 85 years old, and skin was involved in all but 1 case (no. 1), while the bone marrow was involved in 3. All cases showed PR, particularly with regard to the skin lesions, except for case no. 4 , in which the skin lesions and gastric invasion of BPDCN disappeared without chemotherapy three months after the initial presentation. However, in this case, the abnormal cells $(13.2 \%)$ in the bone marrow turned out to be blasts of concomitant MDS, which transformed into AML-M5b after one month. Case no. 3 took a peculiar clinical course, whereby the patient exhibited spontaneous PR after mild atraumatic splenic rupture, with an elevation of CRP. Chromosome analyses were available in three cases (no. 3 from the article, no. 1 from a personal communication, and no. 5 from the present case), all of which showed a normal karyotype. With regard to the triggers of remission, two cases (no. 3 and 5) had events that increased the CRP levels, two cases (no. 1 and 4) had no particular events (personal communication), and the details were unknown in the remaining case. Curiously, none of the cases, except for the present case, obtained CR following sepsis. The present case was the first in which remission was observed in bone marrow lesions after 
Table. Reported Cases of Spontaneous Remission of BPDCN.

\begin{tabular}{|c|c|c|c|c|c|c|c|c|c|c|}
\hline $\begin{array}{l}\text { Case } \\
\text { No. }\end{array}$ & $\begin{array}{l}\text { Age }(y) / \\
\text { Sex }\end{array}$ & $\begin{array}{c}\text { Sites of } \\
\text { Involvement }\end{array}$ & Karyotype & Phenotype & $\begin{array}{c}\text { Peripheral } \\
\text { WBC } \\
\left(10^{9} / \mathrm{L}\right)\end{array}$ & $\begin{array}{l}\text { Response } \\
\text { (month) }\end{array}$ & $\begin{array}{c}\text { Event } \\
\text { before CR/ } \\
\text { PR }\end{array}$ & $\begin{array}{l}\text { Second } \\
\text { treatment } \\
\text { (outcome) }\end{array}$ & $\begin{array}{l}\text { Survival } \\
\text { (mo) }\end{array}$ & References \\
\hline 1 & $15 / F$ & $\mathrm{LN}, \mathrm{BM}$ & $46, X Y^{a}$ & CD4, CD56 & 10.6 & PR & $\mathrm{NP}^{\mathrm{a}}$ & $\begin{array}{l}\text { Ara-C, VP-16, } \\
\text { MIT (alive) }\end{array}$ & 18 & 16 \\
\hline 2 & $67 / \mathrm{M}$ & $\begin{array}{c}\text { Skin, } \\
\text { Stomach }\end{array}$ & $\mathrm{NE}^{\mathrm{a}}$ & $\begin{array}{l}\text { CD4, CD56, } \\
\text { CD123 }\end{array}$ & 6 & $\mathrm{CR}$ & $\mathrm{NP}^{\mathrm{a}}$ & $\begin{array}{c}\text { None } \\
\text { (discharged) }\end{array}$ & $\mathrm{NE}^{\mathrm{a}}$ & 17 \\
\hline 3 & 79/M & Skin & NA & CD4, CD56 & NA & PR & NP & NA & NA & 18 \\
\hline 4 & $78 / \mathrm{M}$ & $\begin{array}{l}\text { Skin, LN, } \\
\text { BM }\end{array}$ & $46, X Y$ & $\begin{array}{l}\text { CD4, CD56, } \\
\text { CD123, TCL1 }\end{array}$ & 13.1 & $\begin{array}{l}\text { 1st PR (2 m) } \\
\text { 2nd PR (5 m) }\end{array}$ & $\begin{array}{l}\text { ASR } \rightarrow \text { the } \\
\text { elevation of } \\
\text { CRP }\end{array}$ & $\begin{array}{l}\text { THP-COP } \\
\text { (dead) }\end{array}$ & 18 & 19 \\
\hline 5 & $85 / \mathrm{M}$ & Skin, BM & $46, X Y$ & $\begin{array}{l}\text { CD4, CD56, } \\
\text { CD123, TCL1 }\end{array}$ & 1.4 & PR (2 m) & Sepsis & None (dead) & 8 & $\begin{array}{l}\text { Present } \\
\text { case }\end{array}$ \\
\hline
\end{tabular}

a) Information obtained from personal communication.

BPDCN: blastic plasmacytoid dendritic cell neoplasm, WBC: while blood cell, CR: complete remission, PR: partial remission, LN: lymph node, BM: bone marrow, NP: nothing particular, ASR: atraumatic splenic rupture, MIT: mitoxantrone, THP-COP: cyclophosphamide, pirarubicin, vincristine and prednisolone, NA: not available, NE: not evaluated

sepsis.

Although we must be cautious in drawing generalizations from only five cases, several points are worth mentioning. First, cases with normal karyotypes are more likely to develop SR than others; most cases of BPDCN have chromosome abnormalities (5-7), and in one study, approximately $60 \%$ of cases with analyzable karyotyping results showed complex karyotypes (22). Second, remission of skin lesions seems to be more likely with this disease than with bone marrow lesions. Indeed, two more cases of SR in the skin lesions were described in the abstract of a paper published in Chinese (23). Third, considering the relatively short history of BPDCN, SR in BPDCN may not be as rare as we speculate owing to the scarce literature.

Positive effects of infections on the course of leukemia have been reported since Eisenlohr reported a case of SR of AML following typhoid infection in 1878 (10). Attempts to induce remission by injecting bacterial toxins were made by some investigators, the most famous of whom was William Coley, who injected mixed extracts of killed $S$. pyogenes and Gram-negative $S$. marcescens into patients with inoperable cancer, which reportedly led to an improvement in $10 \%$ of cases (24). Recently, Rashidi reviewed 46 reported cases of AML with SR (12). A fever occurred in $91.3 \%$ of cases before remission, which was largely due to pneumonia $(54.5 \%)$ and bacteremia $(24.2 \%)$. The causative agents included Staphylococcus and Streptococcus, but the pathogens were not identified or described in two-thirds of the patients. Interestingly, the results of chromosome analyses were available in 23 cases, 9 of which showed a normal karyotype, 5 showed $\mathrm{t}(8 ; 21)(\mathrm{q} 22 ; \mathrm{q} 22)$, and only 3 showed complex abnormalities. This preferential occurrence of prognostically favorable karyotypes, including normal karyotypes, is in agreement with our review of cases of SR in BPDCN. Another interesting point is that half of the previously presented cases were acute monocytic leukemia (M4/M5); the immunophenotypic overlap between BPDCN and monocytic leukemia is well-known, and Takiuchi et al. reported a case of BPDCN, defined by the positive expression of CD123 and BDCA-2, which exhibited monocyte-like properties, including positive staining with alpha-naphthyl butyrate esterase, supporting the close relationship between the progenitors of monocytes and plasmacytoid dendritic cells (25). Regarding the origin of BPDCN, though, a hypothesis that BPDCN cells and myeloid blast cells have a common origin at the very early stage of differentiation has even been proposed (26), as some cases of BPDCN are associated with myeloid disorders, such as case no. 2 in Table.

The precise mechanisms by which severe infections induce SR remain unknown, but recent studies have revealed that both innate and adaptive immunity are involved in the inhibition of tumor growth during sepsis. During sepsis, pathogen-associated molecular patterns (PAMPs) that are expressed by microbes, as well as damage-associated molecular patterns (DAMPs) released from injured tissues, are recognized by pattern recognition receptors (PRRs), such as the Toll-like receptors (TLR) on various immune cells ( $\mathrm{T}$ lymphocytes, dendritic cells and neutrophils). This results in the activation of the transcription factor NF- $\kappa B$, which is responsible for the production of proinflammatory cytokines, such as IL-1, TNF- $\alpha$ and IL-12. These cytokines induce the maturation of primarily dendritic cells, which are crucial for the initiation of a cytotoxic T-cell mediated antitumor response $(13-15,27)$. Furthermore, the involvement of natural killer (NK) cells in a TLR4-dependent manner in sepsisinduced tumor inhibition was demonstrated in a mouse model (28). In addition, polyclonal hypergammaglobulinemia peaking at the time of leukemic regression has been documented in a few cases and may be due to antibodies made by $\mathrm{B}$ lymphocytes triggered by cytotoxic $\mathrm{T}$ lymphocytes recognizing leukemic blasts (29). Finally, fever-like temperatures are known to induce dendritic cell maturation through the induction of heat shock protein (HSP) 90 (30).

It is interesting that $S$. marcescens was the cause of sepsis in the current case. Historically, Coley's toxin consisted of killed S. pyogenes and S. marcescens, and the addition of 
Serratia has been reported to be crucial $(9,24)$. In this century, Beatriz et al. reported that prodigiosin, a natural red pigment isolated from $S$. marcescens, induces apoptosis in leukemic cells with no marked toxicity in nonmalignant cells in vitro, raising the possibility of its therapeutic use as an antineoplastic drug (31). Recently, Sam et al. reported that the inhibitory effects of prodigiosin on MMP-9 and survivin expression in acute lymphoblastic leukemia (ALL) cells, as well as its pro-apoptotic capacity, represent a novel therapeutic avenue against ALL cells (32). There might be some bacteria-specific effects on the induction of SR in hematological malignancies.

In conclusion, we herein report a case of spontaneous regression of BPDCN after severe sepsis induced by $S$. marcescens. SR of BPDCN may not be quite as rare as is generally thought, particularly in cases with normal karyotypes. Since BPDCN occurs mainly in elderly patients, physicians should be aware of this phenomenon when deciding on treatment choices.

This case report was approved by the Ethics Committee of Tohoku Medical and Pharmaceutical University.

\section{The authors state that they have no Conflict of Interest (COI).}

\section{Acknowledgement}

We thank Drs. Hajime Yasuda and Norio Komatsu (Juntendo University), and Keiko Hashikawa and Koichi Ohshima (Kurume University) for providing us with clinical information on the patient. We also thank Ms. Chisaki Itoh (Department of Clinical Laboratory, Tohoku Medical and Pharmaceutical University Hospital) and Ms. Kinue Utsumi (Division of Hematology and Rheumatology, Tohoku Medical and Pharmaceutical University Hospital) for their technical assistance.

\section{References}

1. Adachi M, Maeda K, Takekawa M, et al. High expression of CD56 (N-CAM) in a patient with cutaneous CD4-positive lymphoma. Am J Hematol 47: 278-282, 1994.

2. Kameoka J, Ichinohasama R, Tanaka $M$, et al. A cutaneous agranular CD2- CD4+ CD56+ "lymphoma": report of two cases and review of the literature. Am J Clin Pathol 110: 478-488, 1998.

3. Chaperot L, Bendriss N, Manches O, et al. Identification of a leukemic counterpart of the plasmacytoid dendritic cells. Blood 97: 3210-3217, 2001

4. Petrella T, Comeau MR, Maynadié M, et al. 'Agranular CD4+ CD56+ hematodermic neoplasm' (blastic NK-cell lymphoma) originates from a population of CD56+ precursor cells related to plasmacytoid monocytes. Am J Surg Pathol 26: 852-862, 2002.

5. Khoury JD. Blastic plasmacytoid dendritic neoplasm. Curr Hematol Malig Rep 13: 477-483, 2018.

6. Sapienza MR, PiLeri A, Derenzini E, et al. Blastic plasmacytoid dendritic cell neoplasm: state of the art and prospects. Cancers 11: 595-610, 2019.

7. Laribi K, Denizon N, Besancon A, et al. Blastic plasmacytoid dendritic cell neoplasm: from origin of the cell to targeted therapies. Biol Blood Marrow Transplant 22: 1357-1367, 2016.

8. O'Regan B, Hirschberg C. Spontaneous remission: an annotated bibliography. The Institute of Noetic Sciences, Petaluma, 1993.

9. Hobohm U. Fever and cancer in perspective. Cancer Immunol Immunother 50: 391-396, 2001.

10. Eisenlohr C. [Splenic, lymphatic and medullary leukemia with multiple cranial nerve palsy]. Virchows Arch 73: 56-73, 1878 (in German).

11. Dock G. The influence of complicating diseases upon leukemia. Am J Med Sci 127: 563-592, 1904.

12. Rashidi A, Fisher SI. Spontaneous remission of acute myeloid leukemia. Leuk Lymphoma 56: 1727-1734, 2015.

13. Jimemez C, Ribera JM, Abad E, et al. Increased serum tumour necrosis factor during transient remission in acute leukaemia. Lancet 341: 1600, 1993.

14. Musto P, D'Arena G, Melillo L, et al. Spontaneous remission in acute myeloid leukaemia: a role for endogenous production of tumour necrosis factor and interleukin-2. Br J Haematol 87: 879-80, 1994.

15. Trof RJ, Beishuizen A, Wondergem AM, et al. Spontaneous remission of acute myeloid leukaemia after recovery from sepsis. Neth J Med 65: 259-262, 2007.

16. Hashikawa K, Niino D, Yasumoto $S$, et al. Clinicopathological features and prognostic significance of CXCL12 in blastic plasmacytoid dendritic cell neoplasm. J Am Acad Dermatol 66: 278-291, 2012.

17. Yasuda H, Takaku T, Tomomatsu J, et al. Spontaneous regression of spontaneous regression of cutaneous blastic plasmacytoid dendritic cell neoplasm followed by acute monocytic leukemia evolving from myelodysplastic syndrome. Intern Med 53: 2717-2720, 2014.

18. Owczarczyk-Saczonek A, Sokołowska-Wojdyło M, Olszewska B, et al. Clinicopathologic retrospective analysis of blastic plasmacytoid dendritic cell neoplasms. Postepy Dermatol Alergol 35: 128138, 2018.

19. Daitoku S, Onimaru M, Tanimoto K, et al. Atraumatic splenic ruptures triggered both remission and death in a single case of blastic plasmacytoid dendritic cell neoplasm. J Clin Exp Hematopathol 59: 40-45, 2019.

20. Cheson BD, Bennett JM, Kopecky KJ, et al. Revised recommendations of the international working group for diagnosis, standardization of response criteria, treatment outcomes, and reporting standards for therapeutic trials in acute myeloid leukemia. J Clin Oncol 21: 4642-4649, 2003.

21. Yigit N, Suarez LF, Roth LG, et al. CD4-negative variant of cutaneous blastic plasmacytoid dendritic cell neoplasm with a novel PBRM1 mutation in an 11-year-old girl. Am J Clin Pathol 147: 453-460, 2017.

22. Sakamoto K, Takeuchi K. Cytogenetics of blastic plasmacytoid dendritic cell neoplasm: chromosomal rearrangements and DNA copy-number alterations. Hematol Oncol Clin North Am 34: 523538, 2020.

23. Xiong J, Chen H, Zhang M, et al. Blastic plasmacytoid dendritic cell neoplasm: a clinicopathologic study of 7 cases. Zhonghua Yi Zue Za Zhi 94: 599-602, 2014.

24. Starnes CO. Coley's toxins in perspective. Nature 357: 11-12, 1992.

25. Takiuchi Y, Maruoka H, Aoki K, et al. Leukemic manifestation of blastic plasmacytoid dendritic cell neoplasm lacking skin lesion: a borderline case between acute monocytic leukemia. J Clin Exp Hematol 52: 107-111, 2012.

26. Xie W, Zhao Y, Cao L, et al. Cutaneous blastic plasmacytoid dendritic cell neoplasm occurring after spontaneous remission of acute myeloid leukemia: a case report and review of literature. Med Oncol 29: 2417-2422, 2012.

27. Delano MJ, Ward PA. The immune system's role in sepsis progression, resolution, and long-term outcome. Immunol Rev 274: 330-353, 2016. 
28. Vigneron C, Mirouse A, Merdji H, et al. Sepsis inhibits tumor growth in mice with cancer through Toll-like receptor 4-associated enhanced Natural Killer cell activity. OncoImmunology 8: e1641391, 2019.

29. Mitterbauer M, Fritzer-Szekeres M, Mitterbauer G, et al. Spontaneous remission of acute myeloid leukemia after infection and blood transfusion associated with hypergammaglobulinaemia. Ann Hematol 73: 189-193, 1996.

30. Basu S, Srivastava PK. Fever-like temperature induces maturation of dendritic cells through induction of hsp90. Int Immunol 15: 1053-1061, 2003.

31. Montaner B, Navarro S, Pique M, et al. Prodigiosin from the su- pernatant of Serratia marcescens induces apoptosis in haematopoietic cancer cell lines. Br J Pharmacol 131: 585-593, 2000.

32. Sam MR, Ghoreishi S. Prodigiosin produced by Serratia marcescens inhibits expression of MMP-9 and survivin and promotes caspase-3 activation with induction of apoptosis in acute lymphoblastic leukaemia cells. J Appl Microbiol 125: 1017-1029, 2018 .

The Internal Medicine is an Open Access journal distributed under the Creative Commons Attribution-NonCommercial-NoDerivatives 4.0 International License. To view the details of this license, please visit (https://creativecommons.org/licenses/ by-nc-nd/4.0/).

(C) 2021 The Japanese Society of Internal Medicine Intern Med 60: 927-933, 2021 